\title{
Precentral Knob Corresponds to the Primary Motor and Premotor Area
}

\author{
N. Shinoura, Y. Suzuki, R. Yamada, Y. Tabei, K. Saito, K. Yagi
}

\begin{abstract}
Purpose: Cortical mapping during awake surgery assesses intraoperative neurological change in response to electrical stimulation to provide direct information regarding the anatomical localization of the primary motor area (M1). The goal of the present study was to analyze the reliability of the identification of the precentral knob in the axial image of magnetic resonance imaging or functional MRI (fMRI) for the detection of M1. Materials and Methods: Among patients with brain tumors within or near M1 in whom awake surgery was employed from April 2004 through March 2007, 14 cases were analyzed in which either the M1 or premotor area (PMA) was successfully detected by mapping during awake surgery. Results: The precentral knob was localized to the PMA in 4 cases and to M1 in 10 cases. By contrast, the gyrus activated by hand clenching in fMRI on the affected side at least partially corresponded to M1 in all cases, while those on the unaffected side corresponded to M1 in 12 of 12 cases. Conclusion: These results indicate that the precentral knob corresponds to PMA as well as to M1, whereas the gyrus activated in fMRI corresponds to M1 on the affected and unaffected side.
\end{abstract}

RÉSUMÉ: Le noeud précentral correspond au cortex moteur et prémoteur primaire. Objectif : La cartographie corticale pendant la chirurgie sous anesthésie locale évalue les changements neurologiques en cours d'intervention en réponse à une stimulation électrique afin de fournir des informations directes sur la localisation anatomique de la zone motrice primaire (M1). Le but de cette étude était d'analyser la fiabilité de l'identification du noeud précentral sur l'image axiale obtenue par imagerie par résonance magnétique (IRM) ou IRM fonctionnelle (IRMf) pour la détection de M1. Matériels et méthodes : Quatorze patients atteints de tumeurs cérébrales dans ou au voisinage de M1 chez qui soit M1 ou l'aire prémotrice (APM) a été détectée avec succès par la cartographie pendant la chirurgie sous anesthésie locale, entre avril 2004 et mars 2007. Résultats : Le noeud précentral a été localisé à l'APM chez 4 cas et à M1 chez 10 cas. Par contre, le gyrus activé par le serrement du poing à l'IRMf du côté atteint correspondait au moins partiellement à M1 chez tous les cas, alors que du côté non atteint c'était M1 chez 12 cas sur 12 . Conclusion : Ces résultats indiquent que le noeud précentral correspond à l'APM ainsi qu'à M1, alors que le gyrus activé par l'IRMf correspond à M1 du côté atteint et du côté non atteint.

Can. J. Neurol. Sci. 2009; 36: 227-233

Identification of the primary motor area (M1) using computerized tomography (CT) or magnetic resonance imaging (MRI) is critical to delineate the effect of various diseases (e.g., brain tumors, infarction, multiple sclerosis) as well as for the preoperative planning for patients undergoing resection of brain tumors. The utility of non-invasive identification of M1 using functional MRI (fMRI) has been demonstrated by comparing the location of M1 detected by fMRI with that by invasive and direct methods, such as intraoperative cortical mapping ${ }^{1-3}$. Recent studies suggest that the precentral knob is an anatomic landmark of the $\mathrm{M} 1^{4-13}$. However, the location of precentral knob has never been compared with M1 as detected by invasive and direct methods, namely cortical mapping during awake surgery. Thus, the goal of the present study was to compare the accuracy of precentral knob, as localized by fMRI, as an anatomic landmark for of M1. 


\section{Patients and Methods}

\section{Patients}

Among the patients with brain tumors in M1 in whom awake surgery was employed from April 2004 through March 2007, the M1 or premotor area (PMA) was successfully detected by mapping during awake surgery in 14 out of 32 patients for brain tumors within or near M1. An fMRI was performed preoperatively to localize M1 ${ }^{14}$. Clinical characteristics of 14 patients (five women, nine men; age range, 47-67 years) undergoing awake surgery are summarized in the Table. Pathology of brain tumors was as follows: 11 metastatic tumors and 3 gliomas. The location of brain tumors was as follows: five tumors within M1, four tumors within premotor area, two tumors at primary sensory area (S1) to M1, one tumor at M1 to S1, and one tumor at M1 to PMA. S1 to M1 indicates that the brain tumor was located mainly in S1, which compressed M1. Similarly, M1 to $\mathrm{S} 1$ indicates that the brain tumor was located mainly in M1, which compressed S1. M1 to PMA indicates that the brain tumor was located mainly in M1, which compressed PMA.

\section{Functional MRI and image analysis}

All studies were performed on a 1.5T General Electric Signa Horizon Lx imager. The fMRI and image analysis was performed as described previously ${ }^{15}$. Briefly, the fMRI acquisition sequence consisted of a gradient echo-EPI TE/TR = $82.5 / 3000 \mathrm{~ms}, 128 \times 128$ matrix, with a $24 \mathrm{~cm} \mathrm{x} 24 \mathrm{~cm}$ field of view and a block design of 30 -second intervals. The reconstructed voxel size was $1.88 \times 1.88 \times 2.3 \mathrm{~mm}^{3}$. Fivemillimeter thickness slices were obtained with 70 repetitions. The patient was asked to perform repetitive closing of the hand

Table: Patient characteristics and M1 localization by mapping, fMRI, and precentral knob

\begin{tabular}{c|c|c|c|c|c|c|c|c|c}
\hline No & Sex & Age & Patho & $\begin{array}{c}\text { Location of } \\
\text { tumor }\end{array}$ & Map & $\begin{array}{c}\text { M1/affected } \\
\text { (Knob vs. } \\
\text { Map) }\end{array}$ & $\begin{array}{c}\text { M1/affected } \\
\text { (fMRl vs. } \\
\text { Map) }\end{array}$ & $\begin{array}{c}\text { Symmetry of M1 } \\
\text { (affected/Map vs. } \\
\text { unaffected/fMRl) }\end{array}$ & $\begin{array}{c}\text { M1/unaffected } \\
\text { (Knob vs. } \\
\text { fMRI) }\end{array}$ \\
\hline 1 & M & 57 & Meta & R.PMA & M1 & D(one gyrus) & A & Symmetrical & D(one gyrus) \\
\hline 2 & M & 49 & Meta & L.PMA & M1 & D(one gyrus) & Partially A & Symmetrical & Partially A \\
\hline 3 & M & 51 & Meta & R.PMA & PMA & D(one gyrus) & A & Symmetrical & D(one gyrus) \\
\hline 4 & $\mathrm{~F}$ & 67 & Meta & R.M1 & PMA & ND & A & Symmetrical & D(one gyrus) \\
\hline 5 & M & 64 & Meta & L.M1 & M1 & A & A & Symmetrical & A \\
\hline 6 & M & 47 & Meta & R.PMA & M1,PMA & A & A & Symmetrical & A \\
\hline 7 & F & 49 & Meta & L.S1-M1 & M1 & A & A & Symmetrical & A \\
\hline 8 & M & 61 & Meta & R.M1-PMA & PMA & A & A & Symmetrical & A \\
\hline 9 & M & 48 & Meta & L.S1 & M1 & A & A & Symmetrical & A \\
\hline 10 & M & 66 & Glioma & R.M1 & M1 & A & A & Symmetrical & A \\
\hline 11 & F & 64 & Meta & L.M1 & M1 & A & A & NP & NP \\
\hline 12 & F & 57 & Meta & L.M1 & M1 & A & A & NP & NP \\
\hline 13 & F & 52 & Glioma & L.S1-M1 & M1 & ND & A & Symmetrical & A \\
\hline 14 & M & 64 & Glioma & L.M1-S1 & M1 & ND & A & Symmetrical & A \\
\hline
\end{tabular}

No: No=patient number, Sex: M=Male, F=Female, Patho: Patho=Pathology of tumor, Meta=Metastasis, Map: Location of primary motor area (M1) or premotor area (PMA) was decided by mapping during awake surgery, Location of tumor: R=Right, L=Left, S1=primary sensory area, S1-M1=the tumor was located mainly at S1, which compressed M1, M1-S1=the tumor was located mainly at M1, which compressed S1, M1-PMA=the tumor was located mainly at M1, which compressed PMA, M1/affected (Knob vs. Map): M1 on the affected side was decided either using precentral knob, namely an inverted omega or horizontal epsilon in axial view of MRI (=Anatomy) or by mapping during awake surgery (=Map). Since mapping is the most reliable method to detect M1, the location of M1 corresponding to the precentral knob was rated as agree (=A) or disagree (=D) with that mapped during awake surgery on the affected side. One gyrus= the precentral knob was located at the precentral sulcus, and thus that anatomical landmark was one gyrus rostral to the M1 (i.e., the PMA). ND= the precentral knob was not detected due to the compression by tumor, M1/affected (fMRI vs. Map): M1 localized by fMRI was rated as agree (=A) with that by mapping during awake surgery. Partially A= location of M1 localized by fMRI partially agreed (=Partially A) with that by mapping during awake surgery, Symmetry of M1 (affected/Map vs. unaffected/fMRI): M1 mapped during awake surgery on the affected side was symmetrical (=Symmetrical) to that by fMRI on the unaffected side. NP=fMRI on the unaffected side was not performed, M1/unaffected (fMRI vs. Knob): M1 localized by fMRI was rated as agree (=A), partially agree (=Partially A) or disagree (=D) with localization by the precentral knob, namely an inverted omega or horizontal epsilon in axial view of MRI on the unaffected side. One gyrus= the precentral knob was located at the precentral sulcus, and thus the precentral knob was one gyrus rostral to M1 (i.e., the PMA). $\mathrm{NP}=\mathrm{fMRI}$ on the unaffected side was not performed. 
to a fist (hand clenching), alternating back and forward swing of arm around the shoulder (shoulder swing), alternating back and forward swing of forearm around the elbow (elbow swing), alternating knee flexion and extension (knee flexion) and alternating foot flexion and extension (foot flexion) at approximately seven days before surgery. The fMRI acquisition time was approximately $3.5 \mathrm{~min}$. Anatomic images were acquired using a 3D-FSPGR sequence with TE $2.4 \mathrm{~ms}$, TR 26.0 ms, flip angle $30^{\circ}$, bandwidth $31.25 \mathrm{kHz}$, image matrix $256 \mathrm{x}$ 256, and slice thickness $2.3 \mathrm{~mm}$. After reconstruction, the EPI images were aligned to correct for head motion and were coregistered with anatomic images. The EPI images were smoothed using isotropic Gaussian kernels of $4 \mathrm{~mm}$ and statistically analyzed using the SPM program ${ }^{16}$. The significance of the activations ( $\mathrm{p}$ value) was less than 0.05 .

\section{Awake tumor resection and detection of the location of M1 or PMA by mapping}

Mapping and awake tumor resection was performed as described previously ${ }^{17}$. Briefly, patients were positioned in the supine position and given rigid head fixation (Sugita headrest; Mizuho Medical Co., Tokyo, Japan) after administration of a local anesthetic agent ( $1 \%$ xylocaine and $0.75 \%$ anapain) at the pin sites and regional field block sites. Under general anesthesia with propofol by laryngeal mask airway, the skin was infiltrated with the same local anesthetic agent and incised, and neuronavigated craniotomy and incision of the dura was performed.

After the removal of the laryngeal airway, oxygen was administered via a mask, and cortical mapping was performed by stimulating the cortex with the modified Ojemann stimulator ${ }^{18}$. To avoid inducing intraoperative seizure, a low-stimulus setting (3-5 mA, 60-Hz biphasic square wave pulse of $1 \mathrm{msec} /$ phase for 4 seconds duration) was used. Of note, we did not use after discharge potentials to identify stimulus spread from the intended site of stimulation. When the patient underwent transient deterioration of motor strength by electrical stimulation, the gyrus which included that lesion was regarded as the $\mathrm{M} 1^{18}$. In addition, when the patient showed involuntary movement of flexion with rigidity, the gyrus which included that lesion was regarded as the PMA. All patients were continuously observed by the neurosurgeon, and movements of tongue swing, eye closing, hand clenching, elbow flexion, knee flexion, and foot flexion were noted and reported to the operator.

Corticotomy was performed, avoiding the sites that were activated by fMRI or that induced paresis by cortical mapping in M1. The tumor was removed in the usual fashion. Continuous adequate task judging from the preoperative fMRI, and intraoperative cortical mapping was performed during the removal of tumor near the eloquent motor area responsible for the movement of hand, arm, leg, eye and speech. Removal of tumor was assisted by a neuronavigation system, which showed the location of the area activated in $\mathrm{fMRI}^{17}$. Gross total resection was attempted in all cases. If mild weakness occurred at any point during the resection, the operation was interrupted, and motor function was assessed over the next five minutes. If the motor function did not recover, the operation was terminated, unless the tumor was large enough to endanger the life of patient.
After closure of the dura, the bone flap was replaced, and the skin was closed in the usual manner.

\section{Correlation between M1 and precentral knob}

The location of the precentral knob (i.e. the inverted omega or horizontal epsilon shape in the axial image of MRI) was evaluated as a anatomic landmark for M1 that was mapped on the affected side. Four neurosurgeons reviewed the axial view of MRI independently to determine the location of the precentral knob, which was concordant in all 14 cases. In addition, to evaluate the reliability of fMRI for the detection of M1 on the affected side, the location of M1 was compared using fMRI and mapping.

Since the area activated by fMRI on the affected side may be altered by functional reorganization in patients with brain tumors, localization of M1 on the unaffected side using fMRI during patient hand clenching was also determined to reliably show the location of $M 1^{14,19}$, as localization of the $\mathrm{M} 1$ is typically symmetrical in the cerebral hemispheres ${ }^{20}$. The reliability of M1 localization was then compared between localization of the precentral knob in the axial view of MRI and fMRI on the unaffected side.

\section{RESULTS}

Relationship between precentral knob and M1 by mapping during awake surgery

In 3 of 14 cases, the PMA, rather than the M1, was detected by mapping, possibly due to the low sensitivity of mapping around the tumor ${ }^{21}$. However, the gyrus caudal to PMA (i.e. namely M1) on the affected side was symmetrical with the M1 on fMRI performed on the unaffected side ${ }^{14}$. In 3 of 11 cases, the M1 corresponding to the precentral knob (black arrow head in Figure 1 and Figure 2A) was one gyrus rostral to the M1 mapped during awake surgery (black arrow in Figure 1 and 2A) on the affected side (Table). In eight cases, M1 the corresponding to the precentral knob (black arrow head in Figure 3) was consistent with the M1 mapped during awake surgery (black arrow in Figure 3 ) on the affected side. However, in four cases, the precentral knob was not detected due to compression by brain tumors (Table).

\section{Relationship between M1 localized by fMRI and MI mapped during awake surgery}

In 13 of 14 cases, the gyrus activated in fMRI was consistent with M1 mapped during awake surgery (black arrow in Figure 1 to 3 ) (Table). In Case 2, two gyri were activated by fMRI with hand clenching on the affected side (Figure 1, Panel B2), and the rostral gyrus was consistent with the M1 mapped during awake surgery (black arrow in Figure 1B). In all 12 cases in which fMRI with hand clenching was performed on the unaffected side, the activated gyrus on the unaffected side (red arrow in Figure 1A, 2, 3) was symmetrical to the M1 mapped during awake surgery (black arrow in Figure 1 to 3) (Table). These results indicate that the gyrus activated by hand clenching in fMRI on the affected side at least partially corresponded to M1 in all cases, while those on the unaffected side corresponded to M1 in all cases 

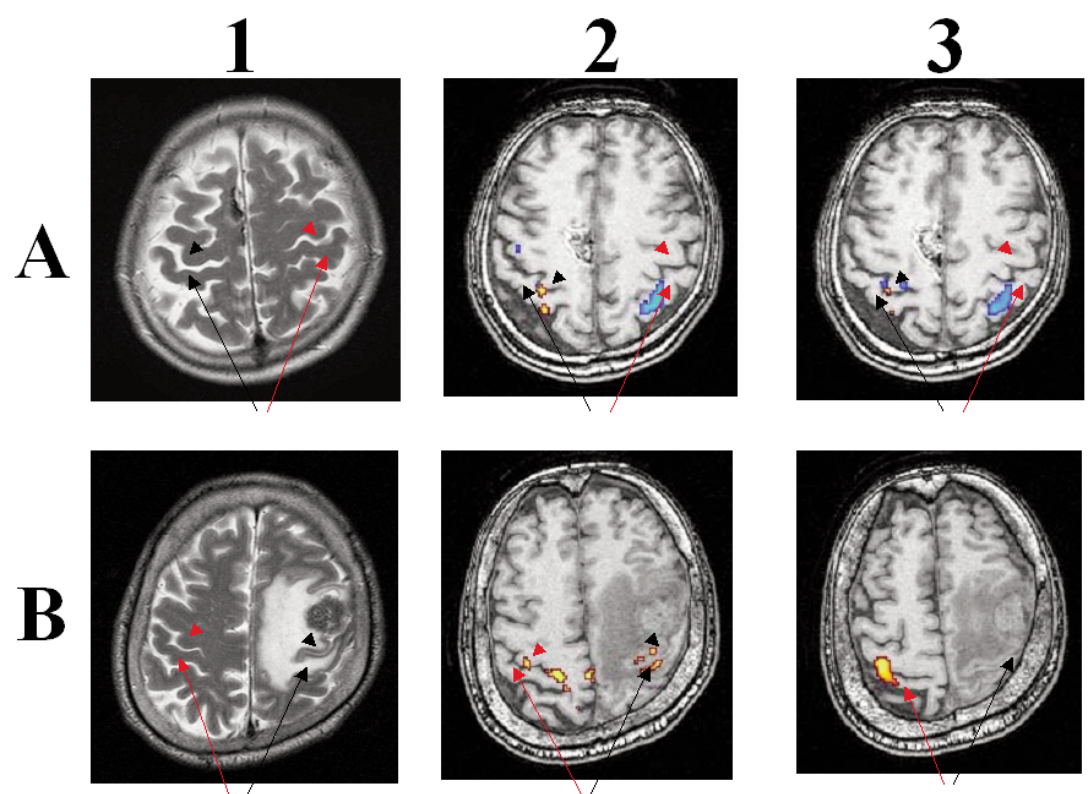

Figure 1: Panel A: Case 1, A 57-year-old male with a metastatic brain tumor located in the right-sided cortex at two gyri rostral to M1. Axial fMRI images constructed during hand clenching (left hand clenching-orange, right hand clenching-blue) are shown (Panel A2, 3). Black arrows indicate M1 mapped during awake surgery on the affected side. Red arrows indicate MI localized by fMRI on the unaffected side. MI on both sides were symmetrical. Arrow heads (black=affected side, red=unaffected side) indicate the anatomical landmark of the precentral knob, namely an inverted omega or horizontal epsilon. The gyrus with the precentral knob was located one gyrus rostral to M1 (i.e. the PMA). Panel B: Case 2, A 49-year-old male with a metastatic brain tumor located in the left-sided cortex at gyrus rostral to M1 (i.e., the PMA). Axial fMRI images constructed during right (Panel B2orange) and left (Panel B3-orange) hand clenching are shown. Black arrows indicate M1 mapped during awake surgery on the affected side. Red arrows indicate M1 on the unaffected side activated by fMRI and symmetrical to M1 of the affected side. Arrow heads (black=affected side, red=unaffected side) indicate the precentral knob, namely an inverted omega. The gyrus with the precentral knob was located one gyrus rostral to M1 (i.e. the PMA). Of note, right hand clenching activated the M1 and PMA of both hemispheres.
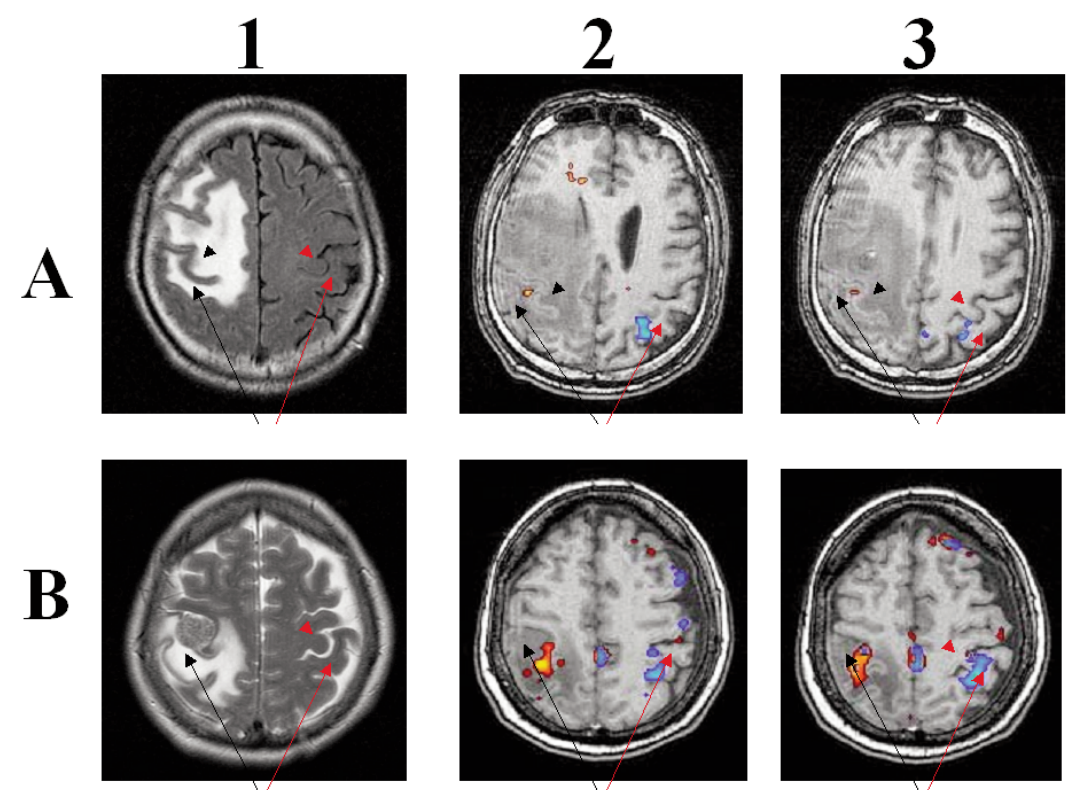

Figure 2: Panel A: Case 3, A 51-year-old male with a metastatic brain tumor located in the right-sided cortex one gyrus rostral to M1 (i.e. the PMA). Axial fMRI images constructed during hand clenching (left hand clenching-orange, right hand clenching-blue) are shown (Panel A2, 3). Black arrows indicate M1 mapped during awake surgery on the affected side. Red arrows indicate M1 localized by fMRI on the unaffected side. M1 on both sides were symmetrical. Arrow heads (black=affected side, red=unaffected side) indicate the precentral knob, namely inverted omega. The gyrus with the anatomical landmark was located one gyrus rostral to M1 (i.e. the PMA). Panel B: Case 4, A 67-year-old female with a metastatic brain tumor located in the right-sided cortex of M1. Axial fMRI images constructed during hand clenching (left hand clenching-orange, right hand clenching-blue) are shown (Panel A2, 3). Black arrows indicate M1 mapped during awake surgery on the affected side. Red arrows indicate M1 localized by fMRI on the unaffected side. M1 on both sides were symmetrical. Red arrow heads indicate the precentral knob, namely the inverted omega, on the unaffected side. The gyrus with the precentral knob was located one gyrus rostral to M1 (i.e. the PMA). 


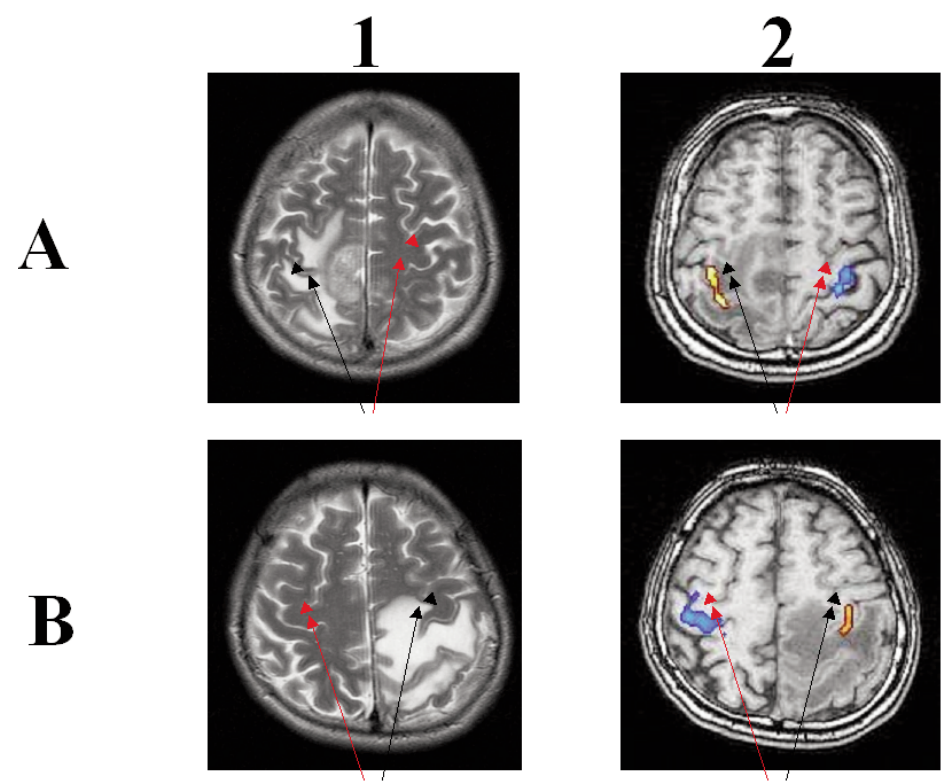

Figure 3: Panel A: Case 8, A 61-year-old male with a metastatic brain tumor located in the right-sided cortex at M1 and PMA. Axial fMRI images constructed during hand clenching (left hand clenching-orange, right hand clenching-blue) are shown (Panel A2). Black arrows indicate M1 mapped during awake surgery on the affected side. Red arrows indicate M1 localized by fMRI on the unaffected side. M1 on both sides were symmetrical. Arrow heads (black=affected side, red=unaffected side) indicate the precentral knob, namely the inverted omega. The gyrus with the precentral knob was consistent with M1 mapped during awake surgery on the affected side and with M1 localized by fMRI on the unaffected side. Panel B: Case 9, A 48year-old male with a metastatic brain tumor located in the left-sided cortex at S1. Axial fMRI images constructed during hand clenching (left hand clenching-orange, right hand clenching-blue) are shown (Panel B2). Black arrows indicate M1 mapped during awake surgery on the affected side. Red arrows indicate M1 localized by fMRI on the unaffected side. M1 on both sides were symmetrical. Arrow heads (black=affected side, red=unaffected side) indicate the precentral knob, namely the inverted omega. The gyrus with the precentral knob is consistent with M1 mapped during awake surgery on the affected side and with M1 localized by fMRI on the unaffected side.

\section{Relationship between M1 corresponding to the precentral knob and M1 determined by fMRI on the unaffected side}

In Cases 1, 3 and 4, the gyrus localized by determination of the precentral knob (red arrow head in Figure 1A, 2) was one gyrus rostral to the gyrus activated by fMRI (red arrow in Figure $1 \mathrm{~A}, 2$ ) (Table). In Case 2, the gyrus localized by determination of the precentral knob (red arrow head in Figure 1B) was also one gyrus rostral to the gyrus activated by fMRI, which was symmetrical to the M1 of affected side (red arrow in Figure 1B) (Table). In Cases 5 to 10,13 and 14, the gyrus localized determination of the precentral knob (red arrow head in Figure 3) was consistent with the gyrus activated by fMRI (red arrow in Figure 3) (Table). These results indicate that in Cases 1 to 4, the gyrus localized by determination of the precentral knob was the PMA (e.g. one gyrus rostral to M1), whereas in cases 5 to 14 , the gyrus localized by determination of the precentral knob was the M1.

\section{DISCUSSION}

The present study using cortical mapping during awake surgery and fMRI to demonstrate that the precentral knob was located at the PMA in 4 of 14 cases. Since brain mapping carries the risk of induction of epilepsy and post-epileptic paresis, which can complicate the use of continuous task monitoring during awake surgery, a relatively low setting (3-5 mA) was used in the present study for bipolar stimulation, although we could have increased the stimulation setting to $8-12 \mathrm{~mA}^{15,21}$. Thus, brain mapping actually localized the M1 in only 14 of 32 cases during awake surgery for brain tumors within or near M1. In these 14 cases, the gyrus detected by brain mapping accurately indicated the location of $M 1^{18,22}$. This is confirmed by demonstration of motor compromise during awake surgery for resection of tumors located in M1 but not during removal of tumors in PMA or S1. Further, localization of M1 by fMRI was consistent with that obtained using mapping during awake surgery (Table). Of note, since tumor perturbation of local anatomy and physiology impact the anatomic localization, fMRI or intraoperative stimulation setting, we utilized localization of M1 on the unaffected side using fMRI during patient hand clenching in order to reliably show the location of M1 on the affected side ${ }^{14,19}$. Based on this determination of M1, we found that electrical stimulation arrested hand clenching on M1, while it elicited involuntary movement of flexion of arm with rigidity on PMA in all 14 cases (Table). Due to this accurate localization of M1, patient outcome did not reflect the results that the precentral knob was located at the PMA in four cases. 
The present study demonstrated that the precentral knob could be used to identify the M1 (ten cases) and PMA (four cases). Yousry et al reported detection of motor hand activation in the precentral gyrus and in the anterior face of the postcentral gyrus ${ }^{3,4}$, possibly due to the mismatch between the anatomical and functional images, existence of pyramidal cells in postcentral gyrus, or activation of sensory afferents by positional changes of hands or fingers. However, since the precentral knob may indicate either the Mlor the PMA, the gyrus, which was activated by fMRI in their study and one gyrus caudal to precentral knob, might be actually M1. The reliability of fMRI is supported by the fact that M1 on both the affected and unaffected side corresponded to M1 by mapping during awake surgery. Therefore, the use of the precentral knob as an anatomic landmark for M1 may not be of utility in patients undergoing surgical resection of tumor within or near M1.

The rate of accurate localization of M1 on the affected side was high with fMRI (e.g. 13 of 14 cases). However, in Case 2, the M1 and PMA were both activated by hand clenching on the affected side. A previous study used quantitative histological and imaging techniques in specimens from autopsy cases to demonstrate that localization of the M1 in the cerebral hemisphere was symmetrical ${ }^{20}$, which is consistent with observations from the present study that M1 localization on the affected and unaffected side by fMRI was symmetrical in 12 of 12 cases. These data enable valid comparisons between M1 localized by the precentral knob and M1 localized on the unaffected side by fMRI. In Cases 1, 3 and 4, the gyrus of precentral knob was one gyrus rostral to M1 (i.e. the PMA) on the unaffected side. The results indicate that the gyrus of the precentral knob corresponded to the PMA in Cases 1 to 4 and to M1 in Cases 5 to 14.

A fMRI has been used in the clinical setting to localize M123,24. The present study demonstrated that fMRI with hand clenching of the unaffected side localized M1 in 12 of 12 cases, based on the principle of symmetry of M1 on the affected and unaffected side. However, in one patient (Case 2), the gyrus activated by fMRI corresponded to the M1 and PMA in both hemispheres, possibly due to the reorganization of motor area in the context of malignancy. This suggests that the gyrus activated by fMRI did not represent M1 alone in some cases (Figure 1B). Therefore, the most reliable non-invasive method of preoperative M1 localization may be via the identification of M1 on the unaffected side by hand clenching in fMRI. It is to be noted that since most of this series represented metastases, these results might not apply as well in glioma patients in which tissue architecture is remodeled and modified, and thus fMRI might not be reliable.

In conclusion, this study indicates that the precentral knob corresponds to PMA as well as to M1, whereas the gyrus activated in fMRI corresponds to M1 on the affected and unaffected side.

\section{ACKNOWLEDGMENTS}

The authors thank H. Shinoura for assistance with manuscript preparation. This work was supported by the Japanese Foundation for Multidisciplinary Treatment of Cancer.

\section{REFERENCES}

1. Jack CR Jr, Thompson RM, Butts RK, Sharbrough FW, Kelly PJ, Hanson DP, et al. Sensory motor cortex: correlation of presurgical mapping with functional MR imaging and invasive cortical mapping. Radiology. 1994;190:85-92.

2. Yetkin FZ, Mueller WM, Morris GL, McAuliffe TL, Ulmer JL, Cox $\mathrm{RW}$, et al. Functional MR activation correlated with intraoperative cortical mapping. AJNR Am J Neuroradiol. 1997; 18:1311-5.

3. Yousry TA, Schmid UD, Jassoy AG, Schmidt D, Eisner WE, Reulen HJ, et al. Topography of the cortical motor hand area: prospective study with functional MR imaging and direct motor mapping at surgery. Radiology. 1995;195:23-9.

4. Yousry TA, Schmid UD, Alkadhi H, Schmidt D, Peraud A, Buettner A, et al. Localization of the motor hand area to a knob on the precentral gyrus, a new landmark. Brain. 1997;120:141-57.

5. Tei H. Monoparesis of the right hand following a localized infarct in the left "precentral knob". Neuroradiology. 1999;41:269-70.

6. Hochman MS, DePrima SJ, Leon BJ. Pseudoulnar palsy from a small infarct of the precentral knob. Neurology. 2000;55: 1939-41.

7. Phan TG, Evans BA, Huston J. Pseudoulnar palsy from a small infarct of the precentral knob. Neurology. 2000;54:2185.

8. Kumabe T, Nakasato N, Inoue T, Yoshimoto T. Primary thumb sensory cortex located at the lateral shoulder of the inverted omega-shape on the axial images of the central sulcus. Neurol Med Chir (Tokyo). 2000;40:393-401.

9. Takahashi N, Kawamura M, Araki S. Isolated hand palsy due to cortical infarction: localization of the motor hand area. Neurology. 2002;58:1412-4.

10. Kim JS, Kwon SU, Lee TG. Pure dysarthria due to small cortical stroke. Neurology. 2003;60:1178-80.

11. Jang SH, Cho SH, Kim YH, Kwon YH, Kwon YH, Byun WM, et al. Cortical activation changes associated with motor recovery in patients with precentral knob infarct. Neuroreport. 2004;15: 395-9.

12. Hanakawa T, Parikh S, Bruno MK, Hallett M. Finger and face representations in the ipsilateral precentral motor areas in humans. J Neurophysiol. 2005;93:2950-8.

13. Hiraga $A$, Ito $S$, Hayakawa $S$, Misawa $S$, Hattori T. Focal shoulder seizures due to an acute small cortical infarction located medial to precentral knob. J Neurol. 2006;253:963-4.

14. Shinoura N, Yamada R, Suzuki Y, Kodama T, Sekiguchi K, Takahashi M, et al. fMRI is more reliable than SEP or mapping for the detection of M1 in proximity to a tumor. Stereotact Funct Neurosurg. 2007;85:99-105.

15. Shinoura N, Suzuki Y, Yamada R, Kodama T, Takahashi M, Yagi K. Fibers connecting the primary motor and sensory area play a role in grasp stability of the hand. Neuroimage. 2005;25:936-41.

16. Friston KJ, Frith CD, Liddle PF, Frackowiak RS. Comparing functional (PET) images: the assessment significant change. J Cereb Blood Flow Metab. 1991;11:690-9.

17. Shinoura N, Yamada R, Kodama T, Suzuki Y, Takahashi M, Yagi K. Preoperative fMRI, tractography and continuous task during awake surgery for maintenance of motor function following surgical resection of metastatic tumor spread to the primary motor area. Minim Invasive Neurosurg. 2005;48:85-90.

18. Berger MS, Kincaid J, Ojemann GA, Lettich E. Brain mapping techniques to maximize resection, safety, and seizure control in children with brain tumors. Neurosurgery. 1989;25:786-92.

19. Shinoura N, Suzuki Y, Yamada R, Kodama T, Takahashi M, Yagi K. Restored activation of primary motor area from motor reorganization and improved motor function after brain tumor resection. AJNR Am J Neuroradiol. 2006;27:1275-82.

20. White LE, Andrews TJ, Hulette C, Richards A, Groelle M, Paydarfar J, et al. Structure of the human sensorimotor system. II: Lateral symmetry. Cereb Cortex. 1997;7:31-47.

21. Shinoura N, Yamada R, Kodama T, Suzuki Y, Takahashi M, Yagi K. Intraoperative cortical mapping has low sensitivity for the detection of motor function in proximity to a tumor in the primary motor area. Stereotact Funct Neurosurg. 2005;83: $135-41$. 
22. Ojemann G, Ojemann J, Lettich E, Berger M. Cortical language localization in left, dominant hemisphere. An electrical stimulation mapping investigation in 117 patients. J Neurosurg. 1989;71:316-26.

23. Atlas SW, Howard RS 2nd, Maldjian J, Alsop D, Dentre JA, Listerud $\mathrm{J}$, et al. Functional magnetic resonance imaging of regional brain activity in patients with intracerebral gliomas: findings and implications for clinical management. Neurosurgery. 1996;38:329-38.

24. Pujol J, Conesa G, Deus J, Vendrfell P, Isamat F, Zannoli G, et al. Presurgical identification of the primary sensorimotor cortex by functional magnetic resonance imaging. J Neurosurg. 1996;84: $7-13$. 